\title{
LAMal: allons-y, maintenant!
}

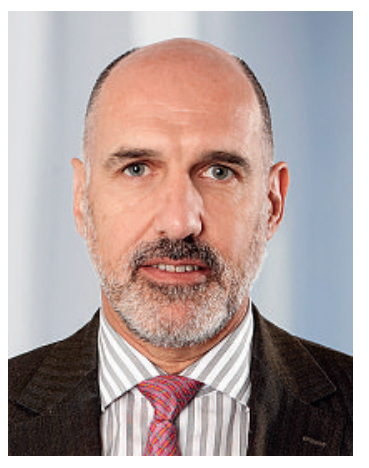

La révision de la LAMal concernant la médecine ambulatoire a fait de grands pas en avant, ces derniers mois; dans leur grande majorité, ces pas vont dans le bon sens. Il s'agit maintenant de régler les derniers points en suspens sans traîner inutilement, respectivement sans donner prise aux manœuvres dilatoires qui apparaissent épisodiquement.

L'un de ces derniers points à régler est la compensation des risques, dont le mauvais fonctionnement actuel conduit tant à la célèbre «chasse aux bons risques» qu'à des «procédures d'économicité» inadéquates des assureurs contre certains collègues: les patients chers représentent un problème qui n'est absolument pas résolu pour le moment. En outre, une compensation des risques affinée sera indispensable dès l'introduction large des réseaux de soins intégrés, pour que les assureurs y participent de façon constructive.

\section{Une compensation des risques affinée, basée sur la morbidité est indispensable dès l'entrée en vigueur de la révision de la LAMal}

Certes, une amélioration de la compensation des risques est prévue pour 2012, mais elle est en réalité insuffisante, voir inadéquate, et trop tardive.

Je m'explique!

Tout d'abord avec la situation des patients chroniques, qui sont ceux qui bénéficient le plus du Managed care. En effet, c'est dans la durée que l'accompagnement «intégré»apporte le plus, sans compter que les réseaux peuvent proposer et rembourser des prestations non-obligatoires dont l'utilité est évidente (diététicienne ou podologue pour les patients diabétiques, par exemple) - il est évident qu'à terme cette prise en charge cohérente, voire élargie, "paie», si l'on ose dire, tant pour les patients concernés que pour le système de santé.

Il faut donc absolument, pour que le Managed care remplisse ses buts, que ces patients lourds soient amenés à y participer. Il faut qu'ils y soient attirés par les prestations proposées et par la qualité de ces dernières, d'une part, mais il faut surtout qu'ils ne soient plus rejetés par les assureurs, voire par les réseaux, parce qu'ils grèvent les budgets. Il faut que ces patients deviennent attractifs! Pour cela, une compensation des risques affinée, basée sur la morbidité (c'est-à-dire sur la charge économique des maladies), est indispensable dès l'entrée en vigueur de la révision de la LAMal.
Autre exemple, les hospitalisations. Au-delà de l'âge et du sexe des assurés, les hospitalisations constituent le nouveau critère de compensation des risques qui doit être pris en compte à partir de 2012 - une hospitalisation fait passer le patient en catégorie «patient cher» et il fait l'objet d'une compensation.

\section{Même si tout n'est pas satisfaisant dans le projet actuel, la révision prévue de la LAMal correspond à beaucoup des points souhaités par la FMH,} et notamment par la Chambre médicale depuis 2006

C'est un exemple typique de fausse bonne idée, l'exemple typique d'un incitatif dangereux! En effet, dans la réalité, il vaut infiniment mieux traiter un patient lourd, un patient cardiaque ou oncologique par exemple, de façon ambulatoire tant que cela reste possible; c'est meilleur pour le patient, et c'est meilleur pour le système de santé. Le problème, c'est qu'avec le système prévu, ce patient ambulatoire n'est pas reconnu comme cher et ne fait pas l'objet d'une compensation ... dans ce sens, il vaudrait mieux l'hospitaliser! Ici de nouveau, une compensation des risques affinée est indispensable dès que possible.

Bref, on l'aura compris, la prise en compte des «critères de morbidité» dans la compensation des risques doit être rapidement introduite. On ne peut pas simplement attendre de voir ce qui se passera après 2012, avec la prise en compte des hospitalisations - l'intégration des critères de morbidité à la compensation des risques doit se faire maintenant, dans le cadre de la révision en cours. Un amendement le prévoyant a été déposé par notre vice-président, le Conseiller national Ignazio Cassis; il doit être soutenu!

Et c'est maintenant l'ensemble de cette révision qui doit aller de l'avant. Quelques tentatives de blocage se font jour en tentant l'introduction dans le projet d'éléments qui conduiraient à un référendum: que ce soit le retour d'une vieille lune, la liberté de contracter, ou que ce soit plus récemment l'obligation du Managed care pour les assurés recevant des subventions pour leurs primes. Ces manœuvres ne sont pas acceptables.

Même si tout n'est pas satisfaisant dans le projet actuel, la révision prévue de la LAMal correspond à beaucoup des points souhaités par la FMH, et notamment par la Chambre médicale depuis 2006. Nous souhaitons que ce projet aboutisse maintenant rapidement. Avec une compensation des risques affinée!

Dr Jacques de Haller, Président de la FMH 